OPEN ACCESS

Edited by: Jiang Chen,

Zhejiang University, China

Reviewed by:

Oronzo Brunetti,

Istituto Nazionale dei Tumori

(IRCCS), Italy

Kazuto Tajiri,

University of Toyama University

Hospital, Japan

${ }^{*}$ Correspondence:

Sasivimol Rattanasir

sasivimol.rat@mahidol.ac.th

Specialty section:

This article was submitted to

Gastrointestinal Cancers,

a section of the journal

Frontiers in Oncology

Received: 15 January 2021

Accepted: 15 March 2021

Published: 31 March 2021

Citation:

Oranratnachai S, Rattanasiri S,

Pooprasert A, Tansawet A,

Reungwetwattana T, Attia $J$ and

Thakkinstian A (2021)

Efficacy of First Line Systemic

Chemotherapy and Multikinase

Inhibitors in Advanced

Hepatocellular Carcinoma:

A Systematic Review and Network

Meta-Analysis.

Front. Oncol. 11:654020.

doi: 10.3389/fonc. 2021.654020

\section{Efficacy of First Line Systemic Chemotherapy and Multikinase Inhibitors in Advanced Hepatocellular Carcinoma: A Systematic Review and Network Meta-Analysis}

\author{
Songporn Oranratnachai ${ }^{1,2}$, Sasivimol Rattanasiri ${ }^{1 *}$, Anantaporn Pooprasert ${ }^{3}$, \\ Amarit Tansawet ${ }^{1,4}$, Thanyanan Reungwetwattana ${ }^{5}$, John Attia ${ }^{6}$ \\ and Ammarin Thakkinstian ${ }^{1}$

\begin{abstract}
Department of Clinical Epidemiology and Biostatistics, Faculty of Medicine Ramathibodi Hospital, Mahidol University, Bangkok, Thailand, ${ }^{2}$ Oncology Clinic, Sriphat Medical Center, Faculty of Medicine, Chiang Mai University, Chiang Mai, Thailand, ${ }^{3}$ Oncology Unit, Division of Internal Medicine, Uttaradit Hospital, Uttaradit, Thailand, ${ }^{4}$ Department of Surgery, Faculty of Medicine Vajira Hospital, Navamindradhiraj University, Bangkok, Thailand, ${ }^{5}$ Division of Medical Oncology, Department of Medicine, Faculty of Medicine Ramathibodi Hospital, Mahidol University, Bangkok, Thailand, ${ }^{6}$ Centre for Clinical Epidemiology and Biostatistics, School of Medicine and Public Health, Hunter Medical Research Institute, University
\end{abstract} \\ of Newcastle, New Lambton, NSW, Australia
}

Background: Hepatocellular carcinoma $(\mathrm{HCC})$ is the third most fatal cancer, with a 5-year survival rate of $18 \%$. Standard frontline-therapy is multikinase inhibitors (MKIs), but accessibility is still limited, particularly in developing countries. This network metaanalysis (NMA) aimed to compare the efficacy of usual chemotherapy vs MKIs.

Method: Randomised-controlled trials (RCTs) comparing any among chemotherapy vs MKIs in treatment-naïve patients with advanced HCCs were identified from MEDLINE and SCOPUS databases. Overall survival (OS) and progression-free survival (PFS) probabilities and times were extracted from Kaplan-Meier curves using Digitizer, and then converted to individual patient time-to-event data. A one-stage mixed-effect survival model was applied to estimate median OS and PFS. A two-stage NMA was applied for the overall response rate and adverse events (AEs) outcome.

Results: A total of 20 RCTs were eligible for NMA. Lenvatinib was the best treatment among single MKIs, with median OS and PFS of 9 and 6.3 months, without significant differences in AEs relative to other MKIs. Median OS and PFS were $0.70(-0.42,1.83)$ and $2.17(1.41,2.93)$ months longer with Lenvatinib than Sorafenib. Among chemotherapy agents, FOLFOX4 had the longest median OS and PFS at 7.9 and 4.3 months, respectively, without significant AEs compared to other chemotherapies. The combination of Sorafenib+Doxorubicin prolonged median OS and PFS to 12.7 and 6.3 months, respectively. 
Conclusion: Use of the MKIs Lenvatinib or Sorafenib as first line systemic treatment for advanced HCC could be beneficial. However, FOLFOX4 might be the optimal choice in a developing country where the health-care budget is limited.

Keywords: chemotherapy, first-line systemic treatment, hepatocellular carcinoma, multikinase inhibitors, network meta-analysis

\section{INTRODUCTION}

Hepatocellular carcinoma (HCC) is the third most common cause of cancer-related death worldwide (1). The common risk factors are chronic hepatitis $\mathrm{B}$ or $\mathrm{C}$ virus infection and cirrhosis from any cause. Treatment of HCC depends on the disease stage, which simultaneously considers liver function, performance status, and tumor burden (2, 3). Early-stage disease usually requires only local treatment, whereas advanced-stage disease may need Multikinase Inhibitors (MKIs) given preserved liver functions; otherwise, supportive care is the only option.

Sorafenib was the first MKI approved by the Food and Drug Administration (FDA) in 2017 as frontline therapy for advanced-stage HCC. Sorafenib increased median overall survival (OS) to 10.7 months compared with 7.9 months in placebo (4). Other MKIs [i.e., Brivanib (5), Sunitinib (6), and Linifanib (7)] were subsequently tested in phase-III trials, but failed to improve OS relative to Sorafenib, until Lenvatinib, approved by the FDA in 2018, was shown to prolong OS to 13.6 months (8).

To date, there are nine systematic reviews (SRs) of frontline treatment options for advanced/unresectable HCC published between 2012-2018 (9-17). Most SRs used direct meta-analysis to compare efficacy between chemotherapy agents or between MKIs, but none compared efficacy of chemotherapy and MKIs (10-17). Of those SRs, only one used network meta-analysis (NMA) (9), including 6 randomised-controlled trials (RCTs) with 4,812 patients, to indirectly compare the efficacy and drug toxicity between 5 MKIs (i.e., Sorafenib, Brivanib, Sunitinib, Linifanib, and Sorafenib+Erlotinib) and placebo. Although MKIs might improve clinical outcomes, the accessibility of these drugs is limited due to high cost. Therefore, we aimed to compare the efficacy and adverse events (AEs) of chemotherapy and MKIs on OS, and progression-free survival (PFS) using a NMA approach.

\footnotetext{
Abbreviation: AE, adverse event; AIC, Akaike's information criterion; CI, confidence interval; FDA, Food and Drug Administration; FOLFOX4, Fluorouracil, Leucovorin, and Oxaliplatin; GEMOX, Gemcitabine and Oxaliplatin; HCC, hepatocellular carcinoma; MKIs, multikinase inhibitors; NCCN, the National Comprehensive Cancer Network; NMA, network metaanalysis; ORR, overall response rate; OS, overall survival; PFS, progression-free survival; PIAF, Cisplatin, Interferon $\alpha-2 b$, Doxorubicin, and Fluorouracil; PRISMA, Preferred Items for Systematic Reviews and Meta-Analysis; RCT, randomised-controlled trial; RECIST, the response evaluation criteria in solid tumors; RoB 2, the Revised Cochrane Risk of Bias tool; RR, risk ratio; SR, systematic review; SUCRA, surface under the cumulative ranking curve; WHO, World Health Organization.
}

\section{METHODS}

This SR and NMA were conducted according to Preferred Reporting Items for Systematic Reviews and Meta-Analyses (PRISMA) and registered to PROSPERO (CRD42019145620). Relevant studies were identified from MEDLINE via PubMed and SCOPUS databases through to $30^{\text {th }}$ November, 2019. The search terms were constructed based on patients (advanced or unresectable HCC), interventions (i.e., chemotherapy and MKIs), and outcomes of interest (see Table 1). Titles and abstracts were screened by one reviewer (SO) then randomly checked by second reviewer (SR). The full texts were then independently selected by two reviewers (SO and SR), and disagreements were resolved through discussion with a third reviewer (ATh). The most recent studies were selected when there were multiple publications. The reference lists were reviewed to identify additional relevant studies.

RCTs were eligible if they met the following criteria: studies in adults with advanced HCC who were treatment-naïve; comparing any pair of chemotherapy agents (e.g., Doxorubicin, Oxaliplatin, Fluorouracil or Capecitabine, Nolatrexed), MKIs (e.g., Sorafenib, Lenvatinib, Brivanib, Sunitinib, Linifanib), or placebo; and had at least one of the following outcomes: OS, PFS, overall response rate (ORR), and AEs.

RCTs were excluded if they compared systemic with nonmedical treatments (e.g., surgery, liver transplant), local treatments (e.g., chemoembolisation, radiotherapy, and hepatic arterial/portal vein chemo infusion) or non-chemotherapy/MKI (e.g., hormonal treatment, tumor vaccine, or gene therapy). If there were in an untranslatable languages, or had insufficient data for pooling after three contact attempts with authors they were also excluded.

\section{Interventions}

Interventions of interest were placebo, chemotherapy (e.g., Doxorubicin, Nolatrexed, combination of Cisplatin, Interferon $\alpha-2 b$, Doxorubicin, and Fluorouracil (PIAF), combination of Fluorouracil, Leucovorin, and Oxaliplatin (FOLFOX4)), monotherapy of MKIs (e.g., Sorafenib, Sunitinib, Brivanib, Linifanib, Dovitinib, Lenvatinib, and Nintedanib), combine chemotherapy with MKIs (e.g., Sorafenib+Doxorubicin, Sorafenib+combination of Gemcitabine and Oxaliplatin (GEMOX)), and combination of MKIs (e.g. Sorafenib+Erlotinib, Sorafenib+Everolimus, and Bevacizumab+Erlotinib).

\section{Outcome of Interests}

Outcome of interest were OS, PFS, ORR, and AEs. OS was defined as time since randomization to death from any cause. 
TABLE 1 | Search terms.

\begin{tabular}{|c|c|c|}
\hline Domain & Search terms for MEDLINE & Search terms for SCOPUS \\
\hline Patients & $\begin{array}{l}\text { (Hepatoma OR "liver cell cancer" OR "liver cancer") AND } \\
\text { (advance OR advanced OR unresectable OR unresected OR } \\
\text { inoperable OR metastasis) }\end{array}$ & $\begin{array}{l}\text { ("hepatocellular carcinoma" OR hepatoma OR "liver cell cancer" OR "liver cancer") } \\
\text { AND (advance OR advanced OR unresectable OR unresected OR inoperable OR } \\
\text { metastasis). }\end{array}$ \\
\hline Interventions & $\begin{array}{l}\text { ("chemotherapy" OR adriamycin OR oxaliplatin OR fluorouracil } \\
\text { OR xeloda OR nolatrexed) OR ("targeted therapy" OR } \\
\text { "multikinase inhibitors" OR nexavar OR lenvima OR sutent OR } \\
\text { brivanib OR linifanib) }\end{array}$ & $\begin{array}{l}\text { (chemotherapy OR adriamycin OR doxorubicin OR oxaliplatin OR fluorouracil OR } \\
\text { xeloda OR capecitabine OR nolatrexed) OR ("targeted therapy" OR "multikinase } \\
\text { inhibitors" OR nexavar OR sorafenib OR lenvima OR lenvatinib OR sutent OR sunitinib } \\
\text { OR brivanib OR linifanib). }\end{array}$ \\
\hline Outcome & $\begin{array}{l}\text { Survival OR (toxicity OR toxicities OR "adverse event" OR } \\
\text { "adverse events" OR drug toxicity) OR response }\end{array}$ & Survival OR (toxicities OR “adverse events") OR response \\
\hline $\begin{array}{l}\text { Filter/ } \\
\text { Limit to }\end{array}$ & $\begin{array}{l}\text { clinical study, clinical trial, comparative study, controlled clinical } \\
\text { trial, randomized controlled trial, observational study }\end{array}$ & article, reviews, conference paper \\
\hline
\end{tabular}

Search terms between each domain will be combined with 'AND' Boolean.

PFS was defined as time since randomization to first occurrence of disease progression or death from cancer-related event. If RCTs $(4,5,7,18-21)$ reported only time to progression which was defined as time since randomization to disease progression, time to progression was used instead of PFS. ORR was defined as the proportion of patients who had a best objective tumor response of complete response or partial response. Tumor response was classified using World Health Organisation (WHO) (22) or the response evaluation criteria in solid tumors (RECIST) (23) criteria according to RCTs' report. AEs were at least one of grade 3 or higher of following AEs as for the National Cancer Institute's Common Terminology Criteria for Adverse Events (24): anemia, neutropenia, thrombocytopenia, diarrhea, hand-foot skin reaction, hypertension, or hyponatremia. If RCTs reported these individual AEs rather than overall $\mathrm{AEs}$, the $\mathrm{AE}$ with the maximum incidence was used.

\section{Data Extraction and Quality Assessment}

Two reviewers (SO and AP) independently extracted the following data: general characteristics (i.e., number of patients, RCT phase, country), patient characteristics (i.e., gender, mean age, percent hepatitis $\mathrm{B} / \mathrm{C}$, Child-Pugh A classification, Barcelona Clinic Liver Cancer stage C, portal vein involvement, extrahepatic spreading), treatment regimens, and outcomes (i.e., complete response and partial response, OS, PFS, and AEs).

The probabilities and times of OS and PFS were also extracted from the Kaplan-Meier curve using Digitizer program (25), and then used to simulate individual patient time-to-event data (26). Any disagreements between the two reviewers were resolved by consensus with a third reviewer (ATh). The main outcome of interest was OS; and secondary outcomes were PFS, ORR, and AEs.

Two reviewers (SO and ATa) used the Revised Cochrane Risk of Bias tool (RoB 2) (27) to assess the quality of RCTs; and any disagreements were resolved by consensus with a third reviewer (Ath).

\section{Statistical Analysis}

Interventions were coded according to treatment as placebo, single-chemotherapy, combined-chemotherapy, combinedchemotherapy with MKIs, single-MKIs, and combined-MKIs. For individual patient time-to-event data for OS and PFS, a one- stage approach using a mixed-effect parametric survival model (28) was applied to obtain relative treatment effects. Appropriate survival distributions (e.g., Weibull, exponential, log-logistic, log-normal, generalized gamma) were assessed using Akaike's Information Criterion (AIC); and the model with the smallest AIC was used.

For dichotomous outcomes (i.e., ORR and AEs), the relative treatment effect, risk ratio (RR), were compared using a twostage NMA. The probability of being the best treatment in maximizing ORR and minimal AEs was assessed using a rankogram and the Surface Under the Cumulative Ranking curve (SUCRA). Transitivity was explored by comparing the distribution of co-variables (e.g., gender, co-morbidity, ChildPugh classification, major vascular invasion, extrahepatic spreading) among comparisons. A comparison-adjusted funnel plot was used to assess publication bias.

All analyses were performed with STATA version 16.0 (29). A two-sided p-value less than 0.05 was considered statistically significant for all tests.

\section{RESULTS}

\section{Characteristics of Included Studies}

A total of 4,662 studies were identified; and reasons for exclusion were reported in the PRISMA flow diagram see Figure 1. The overall risk of bias was low at 75\%; see Figure 2. Inter-observer agreement between reviewers was high for both data extraction $($ kappa $=0.96)$ and risk of bias assessment (kappa $=0.88)$.

Twenty RCTs with 7,846 patients were included in the NMA $(4-8,18,19,21,30-40)$. Most were conducted in Asia (40\%), in male patients (76.0\% - 91.5\%), with Child-Pugh A disease except for one study focusing on Child-Pugh B disease (33). The mean age was 49.3 to 65.6 years. A network map for each outcome was constructed see Figure 3, and the characteristics of the included RCTs are shown in more detail in Table 2.

\section{Overall Survival}

Nineteen RCTs $(4-8,18,19,21,30,32-40)$ assessed OS with 16 treatment regimens, see Figure 3A. Sorafenib+GEMOX was excluded due to the Kaplan-Meier curve not being reported. One-stage NMA with a mixed-effect accelerated failure time 


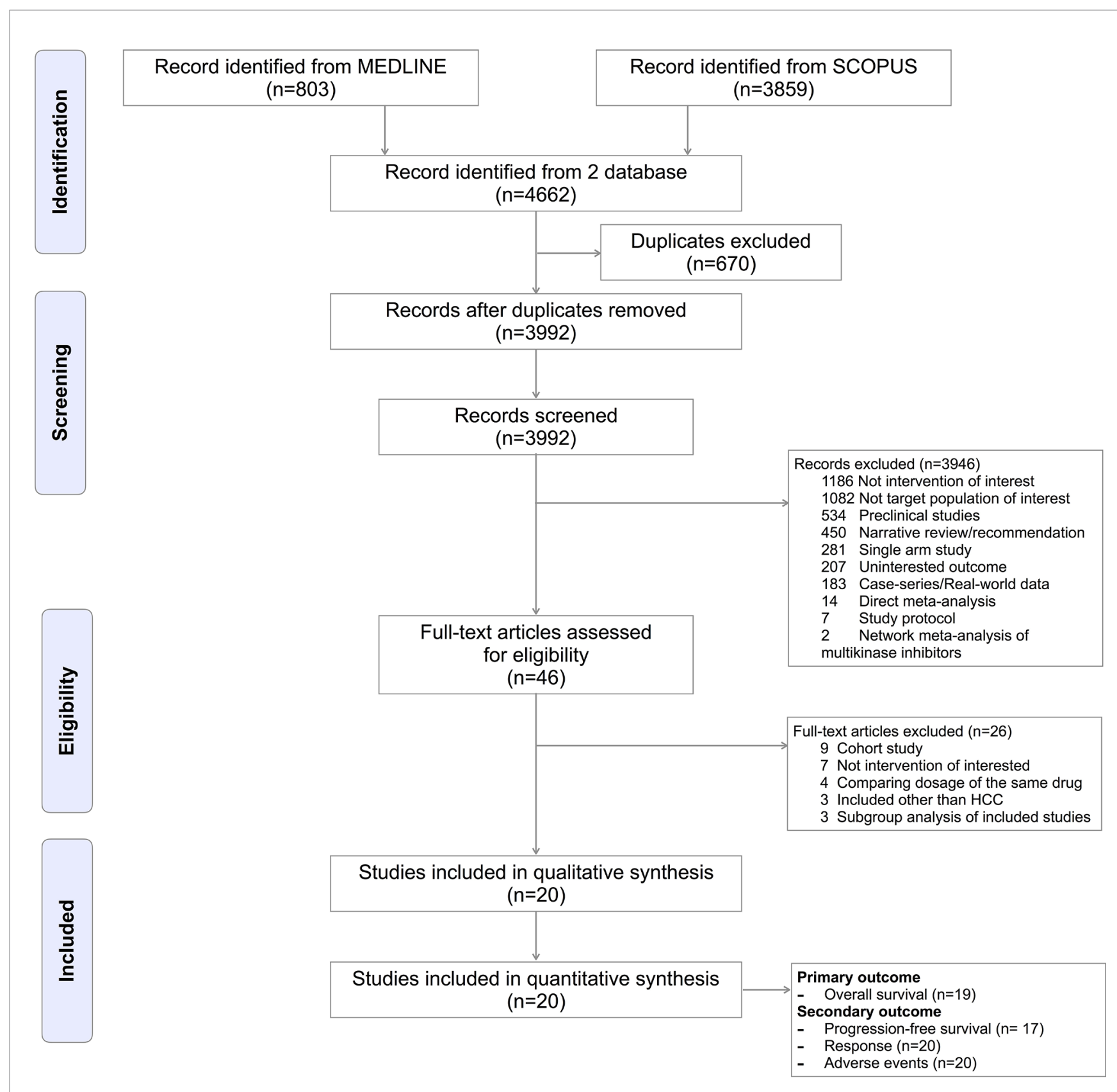

FIGURE 1 | PRISMA flow diagram.

model with log-normal distribution see Table 3, and 5000replication bootstrap was applied to estimate the median OS of each treatment, see Table 4. Among single-chemotherapy regimens, the median OS of Doxorubicin was a month longer than Nolatrexed, but this was not significant (6.9 versus 5.9 months), whereas the median OS of PIAF and FOLFOX4 were not much different (i.e., 7.3 versus 7.9 months). Median OS of single-MKI regimens varied from 6.7 to 9.0 months, in which Lenvatinib/Nintedanib and Sunitinib had the longest and shortest median OSs respectively. Combined-MKI regimens showed some additional benefit with Sorafenib+Doxorubicin having a median OS of 12.7 months. All relative treatment effects by median OS were then compared among active treatments and placebo/no-treatment see Table 5. None of the chemotherapy agents was statistically significantly different when compared to placebo, with differences in median OS ranging from -0.2 to 1.8 months. However, six of seven single-MKIs were significantly different to placebo, prolonging median OS by 0.6 to 3.0 months. Among combination regimens, Sorafenib+Erlotinib and Sorafenib+Doxorubicin, showed median OS (95\% confidence interval (CI)) longer than placebo by 2.55 (1.04, $4.05)$ and $6.62(0.92,12.33)$ months, respectively. Comparing 


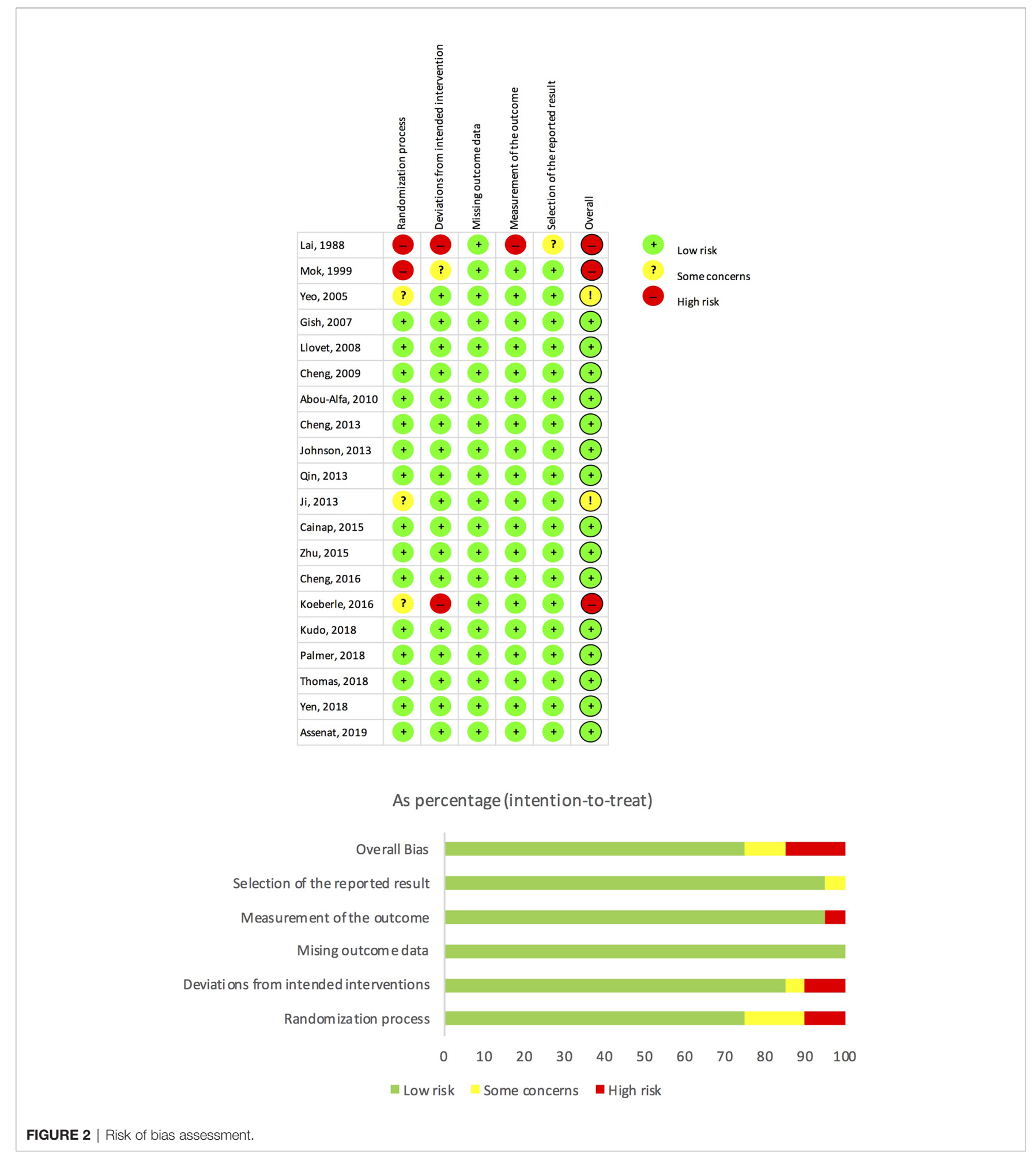

single-MKIs to each other, Sorafenib and Lenvatinib improved median OS by $1.62(0.80,2.44)$ and $2.32(0.93,3.71)$ months, respectively compared to Sunitinib.

Considering current common treatments (i.e., Doxorubicin, FOLFOX4, Sorafenib, and Lenvatinib), Lenvatinib showed the longest OS followed by Sorafenib, FOLFOX4, and Doxorubicin with median OS times of 9.0, 8.3, 7.9, and 6.9 months, respectively. Lenvatinib and Sorafenib showed significantly longer OSs than Doxorubicin. Among chemotherapy agents, FOLFOX4 had the longest OS, and was statistically significant when compared to Nolatrexed (2.01 months, 95\% CI of $0.23,3.80)$. 
A Overall survival

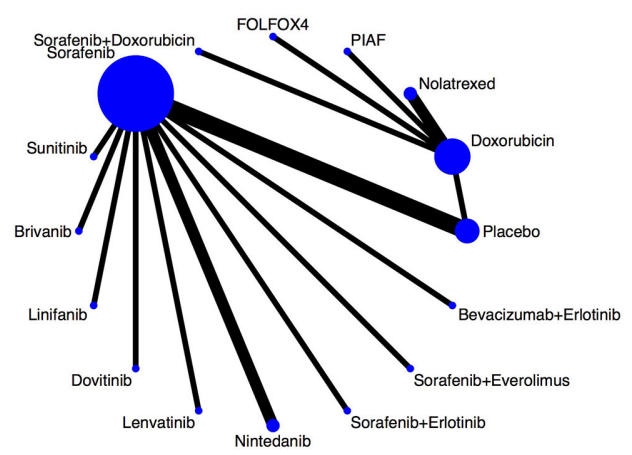

C Overall response rate

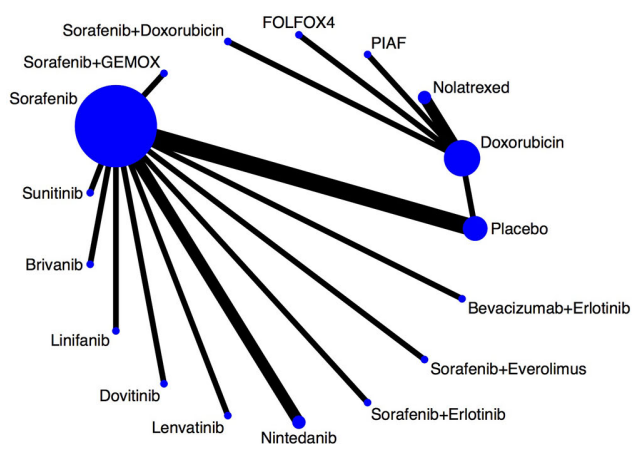

B Progression-free survival

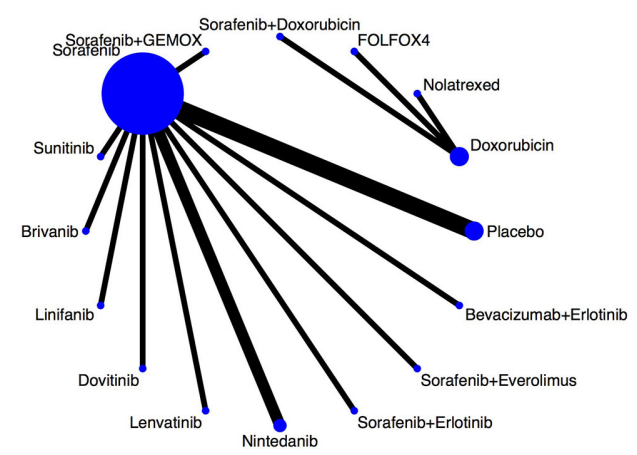

D Adverse events

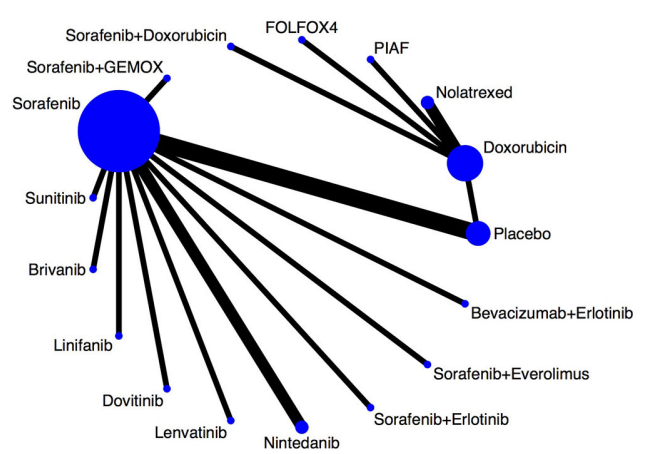

FIGURE 3 | Network map for each outcome. (A - overall survival, B - progression-free survival, C - overall response rate, and D - adverse events).

\section{Progression-Free Survival}

Seventeen RCTs $(4-8,18,19,21,30-33,36-39)$ with 16 regimens were included in the NMA of PFS, see Figure 3B. Median PFS varied from 3.0 to 6.3 months, see Table 4. Comparisons of median PFS indicated all regimens had significantly longer PFSs compared with placebo, except Doxorubicin, Nolatrexed, Dovitinib, Nintedanib, and Sorafenib+Erlotinib, see Table 6. Among chemotherapy agents, only FOLFOX4 had $1.31(0.58$, 2.03) months significantly longer PFS than placebo. Among single-MKIs, Lenvatinib had significantly prolonged PFS of 1.2 to 2.8 months when compared to other agents. Also, Lenvatinib could significantly prolong PFS by 2.0 to 3.1 months when compared with other chemotherapy agents.

Among combined-regimens, Sorafenib+Doxorubicin, Sorafenib+GEMOX, Sorafenib+Everolimus, and Bevacizumab +Erlotinib had median PFS 3.27 (1.12, 5.41), 1.57 (0.03, 3.11), $1.72(0.09,3.36)$, and $2.53(0.60,4.45)$ months significantly longer than placebo.

\section{Overall Response Rate}

Twenty RCTs $(4-8,18,19,21,30-40)$, with 17 regimens were included to estimate RRs (95\% CI) of ORR see Figure 3C and
Table 7. Among chemotherapy agents, FOLFOX4 showed 3.05 $(1.13,8.22)$ and $8.93(1.52,52.59)$ times significantly higher ORR than Doxorubicin and Nolatrexed respectively. PIAF demonstrated $5.84(1.13,30.25)$ times significantly higher ORR than Nolatrexed. Among MKIs, Lenvatinib, Linifanib, and Brivanib showed 9.04 (2.63, 31.07), 5.15 (1.48, 17.90), and 4.18 $(1.23,14.17)$ times significantly higher ORR than placebo, whereas the other MKIs were not significant. Also, Lenvatinib was significantly superior to Sorafenib, Sunitinib, Brivanib, Dovitinib, and Nintedanib with RRs of 2.89 (1.96, 4.26), 2.66 (1.45, 4.85), 2.16 (1.29, 3.64), $5.14(1.68,15.74)$, and 4.59 (1.77, 11.93), respectively. Furthermore, Lenvatinib showed 1.71 (0.80, $3.61)$ and $1.81(0.53,6.12)$ times higher ORR than Sorafenib +Erlotinib and Bevacizumab+Erlotinib, but lower ORR than Sorafenib+Everolimus, FOLFOX4, and PIAF, although none of these was significant. The SUCRAs indicated that the highest ranked treatments were Sorafenib+Everolimus followed by FOLFOX4 (Table 7).

\section{Adverse Events}

Twenty RCTs (4-8, 18, 19, 21, 30-40) with 17 regimens were included to estimated RR (95\% CI) of AEs see Figure 3D and 
TABLE 2 | Characteristics of included studies.

\begin{tabular}{|c|c|c|c|c|c|c|c|c|c|c|c|c|c|}
\hline Author, Year & Treatment & $\mathbf{n}$ & RCT Phase & Region & $\%$ Male & Mean age & $\% \mathrm{HBV}$ & $\% \mathrm{HCV}$ & $\%$ Child A & $\% B C L C$ C & $\% \mathrm{PVI}$ & $\%$ EHS & Outcomes \\
\hline Lai (34) & Doxorubicin vs no treatment & 106 & 3 & AP & 86.8 & 51.4 & 73.6 & - & - & - & - & - & OS, ORR, AEs \\
\hline Mok (35) & Nolatrexed vs Doxorubicin & 54 & 2 & $\mathrm{AP}$ & 90.7 & 54.3 & 77.8 & 5.6 & - & - & 48.1 & 31.5 & OS, ORR, AES \\
\hline Yeo et al. (40) & PIAF vs Doxorubicin & 188 & 3 & - & 91.5 & 49.3 & 81.0 & 6.0 & 85.1 & - & 48.5 & - & OS, ORR, AEs \\
\hline Gish (32) & Nolatrexed vs Doxorubicin & 445 & 3 & US Eu Af & - & - & 22.2 & 39.3 & 73.9 & - & - & _- & OS, PFS, ORR, AES \\
\hline Llovet (4) & Sorafenib vs placebo & 602 & 3 & US Eu AusNZ & 87.0 & 65.6 & 18.4 & 28.1 & 96.5 & 82.4 & 38.4 & 51.3 & OS, PFS, ORR, AES \\
\hline Cheng (18) & Sorafenib vs placebo & 226 & 3 & $A P$ & 85.4 & 52.5 & 73.0 & 8.4 & 97.3 & 95.6 & 35.4 & 68.6 & OS, PFS, ORR, AES \\
\hline Abou-Alfa et al. (30) & Sorafenib+Doxorubicin vs Doxorubicin & 96 & 2 & US EU AP & 76.0 & 62.6 & 10.4 & 17.7 & 97.9 & - & 30.2 & 58.3 & OS, PFS, ORR, AES \\
\hline Cheng et al. (6) & Sunitinib vs Sorafenib & 1074 & 3 & AP & 83.3 & 55.1 & 53.8 & 21.6 & 99.6 & 85.3 & 32.1 & - & OS, PFS, ORR, AES \\
\hline Johnson et al. (5) & Brivanib vs Sorafenib & 1155 & 3 & US Eu AP AusNZ & 83.7 & 57.8 & 44.3 & 20.3 & 92.0 & 77.3 & 19.3 & 49.7 & OS, PFS, ORR, AES \\
\hline Qin et al. (37) & FOLFOX4 vs Doxorubicin & 371 & 3 & $A P$ & 88.7 & 49.4 & 91.4 & 6.7 & 87.9 & 80.1 & - & 58.2 & OS, PFS, ORR, AES \\
\hline Ji et al. (33) & Sorafenib vs no treatment & 189 & 3 & $\mathrm{AP}$ & 84.1 & 59.0 & 84.1 & 2.6 & 0.0 & 87.8 & - & - & OS, PFS, ORR, AES \\
\hline Cainap et al. (7) & Linifanib vs Sorafenib & 1035 & 3 & US Eu AP AusNZ & 85.0 & 56.6 & 53.2 & 25.0 & 94.4 & 82.2 & 43.4 & 58.3 & OS, PFS, ORR, AES \\
\hline Zhu et al. (19) & Sorafenib+Erlotinib vs Sorafenib & 720 & 3 & US EU AP & 80.7 & - & 35.4 & 26.5 & 97.4 & 85.0 & 40.4 & 58.9 & OS, PFS, ORR, AES \\
\hline Cheng et al. (20) & Dovitinib vs Sorafenib & 165 & 2 & $\mathrm{AP}$ & 84.8 & 55.5 & - & - & 99.4 & 97.6 & - & - & OS, PFS, ORR, AES \\
\hline Koeberle et al. (21) & Sorafenib+Everolimus vs Sorafenib & 105 & 2 & - & 83.8 & 62.2 & 17.1 & 28.6 & 82.9 & 72.4 & 30.5 & 55.2 & OS, PFS, ORR, AES \\
\hline Kudo et al. (8) & Lenvatinib vs Sorafenib & 954 & 3 & US EU AP & 84.0 & 58.0 & 50.0 & 23.0 & 99.0 & 79.0 & 21.0 & 61.0 & OS, PFS, ORR, AES \\
\hline Palmer et al. (36) & Nintedanib vs Sorafenib & 93 & 2 & $\mathrm{Eu}$ & 79.6 & 61.5 & 11.8 & 22.6 & 98.9 & 73.1 & 33.3 & 65.6 & OS, PFS, ORR, AES \\
\hline Thomas et al. (38) & Bevacizumab+Erlotinib vs Sorafenib & 90 & 2 & US & - & 61.8 & - & - & 85.6 & 66.7 & 21.1 & 33.3 & OS, PFS, ORR, AES \\
\hline Yen et al. (39) & Nintedanib vs Sorafenib & 95 & 2 & AP & 87.4 & 58.5 & 63.2 & 15.8 & 99.0 & 87.4 & 42.1 & 68.4 & OS, PFS, ORR, AES \\
\hline Assenat et al. (31) & Sorafenib+GEMOX vs Sorafenib & 83 & 2 & $\mathrm{Eu}$ & 89.2 & 62.0 & 3.6 & 15.7 & - & 85.5 & 26.5 & 68.7 & PFS, ORR, AEs \\
\hline
\end{tabular}

AEs, adverse events; Af, Africa; AP, Asia-Pacific; AusNZ, Australia and New Zealand; BCLC C, Barcelona Clinic Liver Cancer stage C; Child A, Child Pugh Classification A; EHS, extrahepatic spreading; Eu, Europe; FOLFOX4, fluorouraci, leucovorin, and oxaliplatin; GEMOX, gemcitabine, oxaliplatin; HBV, hepatitis B viral infection; HCV, hepatitis C viral infection; ORR, overall response rate; OS, overall survival; PFS, progression-free survival; PIAF, cisplatin, interferon $\alpha$-2b, doxorubicin, and fluorouracil; PVI, portal vein involvement; RCT, Randomised-controlled Trial; US, United States. 
TABLE 3 | Mixed effect parametric survival models using different distributions of survival time.

\begin{tabular}{|c|c|c|c|c|c|c|}
\hline Outcome & Survival distribution & Exponential & Weibull & Log-normal & Log-logistic & Gamma \\
\hline \multirow[t]{2}{*}{ OS } & Log likelihood & -19415.874 & -19177.68 & -18883.637 & -18951.691 & -19090.474 \\
\hline & AIC & 38865.75 & 38391.36 & 37803.27 & 37939.38 & 38216.95 \\
\hline \multirow[t]{2}{*}{ PFS } & Log likelihood & -15103.572 & -14908.65 & -14205.728 & -14318.184 & -14742.737 \\
\hline & AIC & 30241.14 & 29853.3 & 28447.46 & 28672.37 & 29521.47 \\
\hline
\end{tabular}

AIC, Akaike's information criterion, OS, overall survival, PFS, progression-free survival.

Table 8. Most grade 3 or higher AEs in chemotherapy were neutropenia, whereas for MKIs, they were diarrhea or hand-foot skin reaction. All regimens except Nolatrexed, Dovitinib, Nintedanib, Sorafenib+Everolimus, and Bevacizumab+Erlotinib had significantly higher AEs compared to placebo, with RRs ranging from 4.99 to 33.83 .

There was no significant difference in AEs among chemotherapy and combined MKI regimens. Only Nintedanib had significantly lower AEs than Sorafenib+GEMOX, Lenvatinib, Brivanib, and Linifanib with RRs of $0.23(0.06,0.92), 0.25(0.08$, $0.85), 0.27(0.08,0.90)$, and $0.30(0.09,0.99)$, respectively. The best ranked regimens in terms of having the least AEs were Nintedanib and Bevacizumab+Erlotinib, see Table 8.

\section{Risk and Benefit Assessment}

Risk (AEs) and benefit (OS) were assessed by estimating the incremental risk-benefit ratio of the treatments recommended by clinical practice guidelines, i.e., Sorafenib, Lenvatinib, and FOLFOX4. Incremental risk-benefit ratios of Lenvatinib and FOLFOX4 versus Sorafenib were jointly simulated for each pair using 1000 Monte Carlo simulations, and then risk of AEs

TABLE 4 | Estimations of median overall survival and median progression-free survival for each treatment regimen.

\begin{tabular}{lcc}
\hline Treatment regimen & $\begin{array}{c}\text { Median OS (95\% Cl) } \\
\text { (months) }\end{array}$ & $\begin{array}{c}\text { Median PFS (95\% Cl) } \\
\text { (months) }\end{array}$ \\
\hline Placebo & $6.08(5.44,6.72)$ & $3.00(2.70,3.29)$ \\
Doxorubicin & $6.91(5.96,7.85)$ & $3.27(2.98,3.55)$ \\
Nolatrexed & $5.88(4.67,7.08)$ & $3.20(2.75,3.66)$ \\
PIAF & $7.25(4.90,9.60)$ & - \\
FOLFOX4 & $7.89(6.22,9.57)$ & $4.30(3.65,4.96)$ \\
Sorafenib + & $12.70(7.08,18.32)$ & $6.27(4.13,8.40)$ \\
Doxorubicin & - & $4.57(3.08,6.05)$ \\
Sorafenib + GEMOX & $8.32(7.74,8.89)$ & $4.09(3.88,4.29)$ \\
Sorafenib & $6.70(5.79,7.61)$ & $3.83(3.40,4.26)$ \\
Sunitinib & $7.64(6.61,8.68)$ & $4.40(3.88,4.91)$ \\
Brivanib & $7.81(6.70,8.93)$ & $5.05(4.40,5.70)$ \\
Linifanib & $8.44(6.08,10.80)$ & $3.57(2.67,4.48)$ \\
Dovitinib & $9.02(7.77,10.27)$ & $6.26(5.48,7.05)$ \\
Lenvatinib & $9.03(6.75,11.33)$ & $3.50(2.63,4.36)$ \\
Nintedanib & $8.63(7.23,10.02)$ & $3.56(3.07,4.05)$ \\
Sorafenib + Erlotinib & $9.37(6.07,12.66)$ & $4.72(3.16,6.28)$ \\
Sorafenib + & & \\
Everolimus & $7.31(4.72,9.90)$ & $5.52(3.63,7.41)$ \\
Bevacizumab + & & \\
Erlotinib & & \\
& & \\
\hline
\end{tabular}

Cl, Confidence Interval; FOLFOX4, fluorouracil, leucovorin, and oxaliplatin; GEMOX, gemcitabine, oxaliplatin; OS, overall survival; PFS, progression-free survival; PIAF, cisplatin, interferon $\alpha-2 b$, doxorubicin, and fluorouracil. and benefit of OS were plotted on $\mathrm{y}$-and $\mathrm{x}$-axis, respectively, see Figure 4.

For Lenvatinib versus Sorafenib, most AEs and OS fell in the right and the left upper quadrants with symmetry about the $\mathrm{x}$ axis, but not in the $y$-axis see Figure $\mathbf{4 A}$; indicating that Lenvatinib offered no benefit in terms of OS, but had higher AEs compared to Sorafenib. FOLFOX4 appears to have lower OS and higher AEs than Sorafenib see Figure 4B.

\section{DISCUSSION}

We conducted a NMA to assess the efficacy of first-line HCC treatments focusing on chemotherapy agents, and MKIs using data from 20 phase II-III RCTs. Our findings suggested that Lenvatinib and FOLFOX4 were the best of the MKIs and chemotherapy agents respectively, prolonging OS and PFS by 9 and 6.3 months and 7.9 and 4.3 months respectively without significantly different AEs relative to other agents in their class. Combining these classes, i.e. Sorafenib+Doxorubicin could prolong OS and PFS to as long as 12.7 and 6.3 months.

Our NMA included all chemotherapy agents and MKIs which had been studied in RCTs up to 2019, including Lenvatinib (8), which was not included in the previous NMA (9). This allowed us to compare the efficacy between chemotherapy and MKIs by borrowing common comparators (e.g., placebo, Sorafenib), which also had not been done before. The previous NMA (9) indicated that Sorafenib, Linifanib, Brivanib, and Sunitinib were significantly better in prolonging OS than placebo, and that the combination of Sorafenib+Erlotinib was the best in prolonging OS, followed by Sorafenib. Our updated findings indicated that Lenvatinib is better than Sorafenib in prolonging OS, PFS, and showed higher ORR. Considering only treatment regimens recommended by the National Comprehensive Cancer Network (NCCN) (41), including Sorafenib, Lenvatinib, and FOLFOX4, our findings showed that Lenvatinib provides the longest OS and PFS with no significant difference in AEs compared to other treatments.

For chemotherapy agents, only direct meta-analysis of Oxaliplatin-based chemotherapy had been published $(12,42)$. The pooled median PFS for Oxaliplatin-based chemotherapy was similar to our FOLFOX4 regimen (4.2 (12) to 4.7 (42) months vs. 4.3 months) but OS was slightly longer than our results (9.3 (12) to 9.4 (42) months vs 7.9 months). The difference in OS may come from heterogeneity in the combination of chemotherapy with Oxaliplatin. 
TABLE 5 | Comparisons of median overall survival among treatment regimens.

\begin{tabular}{|c|c|c|c|c|c|c|c|c|c|c|c|c|c|c|c|}
\hline Placebo & $\begin{array}{c}0.83 \\
(-0.35,2.01) \\
\text { Doxorubicin }\end{array}$ & $\begin{array}{c}-0.20 \\
(-1.62,1.22) \\
-1.03 \\
(-2.14,0.08) \\
\text { Nolatrexed }\end{array}$ & $\begin{array}{c}1.18 \\
(-1.31,3.67) \\
0.35 \\
(-1.93,2.62) \\
1.38 \\
(-1.14,3.90) \\
\text { PIAF }\end{array}$ & $\begin{array}{c}1.81 \\
(-0.02,3.65) \\
0.98 \\
(-0.40,2.37) \\
2.01 \\
(0.23,3.80) \\
0.64 \\
(-2.02,3.30) \\
\text { FOLFOX4 }\end{array}$ & $\begin{array}{c}6.62 \\
(0.92,12.33) \\
5.79 \\
(0.23,11.35) \\
6.82 \\
(1.13,12.51) \\
5.44 \\
(-0.58,11.47) \\
4.81 \\
(-0.94,10.55) \\
\text { Sorafenib+ } \\
\text { Doxorubicin }\end{array}$ & $\begin{array}{c}2.24 \\
(1.46,3.03) \\
1.41 \\
(0.07,2.75) \\
2.44 \\
(0.92,3.96) \\
1.06 \\
(-1.49 .3 .62) \\
0.43 \\
(-1.52,2.37) \\
-4.38 \\
(-10.13,1.38) \\
\text { Sorafenib }\end{array}$ & $\begin{array}{c}0.62 \\
(0.92,12.33) \\
-0.21 \\
(-1.70,1.29) \\
0.82 \\
(-0.85,2.49) \\
-0.55 \\
(-3.18,2.07) \\
-1.19 \\
(-3.25,0.87) \\
-6.00 \\
(-11.76,-0.24) \\
-1.62 \\
(-2.44,-0.80) \\
\text { Sunitinib }\end{array}$ & $\begin{array}{c}1.57 \\
(0.39,2,75) \\
0.74 \\
(-0.85,2.32) \\
1.77 \\
(0.02,3.51) \\
0.39 \\
(-2.27,3.05) \\
-0.25 \\
(-2.38,1.88) \\
-5.05 \\
(-10.87,0.76) \\
-0.67 \\
(-1.60,0.25) \\
0.94 \\
(-0.28,2.17) \\
\text { Brivanib }\end{array}$ & $\begin{array}{c}1.74 \\
(0.48,2.99) \\
0.91 \\
(-0.72,2.53) \\
1.93 \\
(0.16,3.71) \\
0.56 \\
(-2.15,3.27) \\
-0.08 \\
(-2.23,2.08) \\
-4.88 \\
(-10.70,0.94) \\
-0.51 \\
(-1.53,0.52) \\
1.11 \\
(-0.16,2.39) \\
0.17 \\
(-1.19,1.53) \\
\text { Linifanib }\end{array}$ & $\begin{array}{c}2.37 \\
(-0.11,4.84) \\
1.53 \\
(-1.14,4.21) \\
2.56 \\
(-0.20,5.33) \\
1.19 \\
(-2.29,4.66) \\
0.55 \\
(-2.47,3.58) \\
-4.25 \\
(-10.47,1.96) \\
0.12 \\
(-2.27,2.52) \\
1.74 \\
(-0.78,4.27) \\
0.80 \\
(-1.74,3.34) \\
0.63 \\
(-1.98,3.24) \\
\text { Dovitinib }\end{array}$ & $\begin{array}{c}2.94 \\
(1.56,4.32) \\
2.11 \\
(0.35,3.87) \\
3.14 \\
(1.25,5.04) \\
1.77 \\
(-1.05,4.59) \\
1.13 \\
(-1.11,3.37) \\
-3.68 \\
(-9.53,2.18) \\
0.70 \\
(-0.42,1.83) \\
2.32 \\
(0.93,3.71) \\
1.38 \\
(-0.76,2.83) \\
1.21 \\
(-0.32,2.74) \\
0.58 \\
(-2.06,3.21) \\
\text { Lenvatinib }\end{array}$ & $\begin{array}{c}2.96 \\
(0.50,5.41) \\
2.13 \\
(-0.54,4.79) \\
3.16 \\
(0.43,5.89) \\
1.78 \\
(-1.64,5.21) \\
1.14 \\
(-1.88,4.17) \\
-3.66 \\
(-9.91,2.58) \\
0.72 \\
(-1.65,3.09) \\
2.34 \\
(-0.14,4.82) \\
1.39 \\
(-1.14,3.92) \\
1.22 \\
(-1.36,3.80) \\
0.59 \\
(-2.75,3.93) \\
0.01 \\
(-2.61,2.63) \\
\text { Nintedanib }\end{array}$ & $\begin{array}{c}2.55 \\
(1.04,4.05) \\
1.72 \\
(-0.13,3.58) \\
2.75 \\
(0.76,4.74) \\
1.37 \\
(-1.48,4.23) \\
0.74 \\
(-1.59,3.06) \\
-4.07 \\
(-10.00,1.86) \\
0.31 \\
(-0.99,1.61) \\
1.93 \\
(0.41,3.45) \\
0.98 \\
(-0.60,2.57) \\
0.82 \\
(-0.84,2.47) \\
0.19 \\
(-2.54,2.91) \\
-0.39 \\
(-2.12,1.33) \\
-0.41 \\
(-3.10,2.29) \\
\text { Sorafenib+ } \\
\text { Erlotinib }\end{array}$ & $\begin{array}{c}3.29 \\
(-0.11,6.69) \\
2.46 \\
(-1.11,6.02) \\
3.49 \\
(-0.14,7.12) \\
2.11 \\
(-2.06,6.28) \\
1.47 \\
(-2.36,5.31) \\
-3.33 \\
(-9.92,3.25) \\
1.05 \\
(-2.26,4.36) \\
2.67 \\
(-0.72,6.05) \\
1.72 \\
(-1.68,5.13) \\
1.55 \\
(-1.90,5.01) \\
0.92 \\
(-3.15,5.00) \\
0.34 \\
(-3.15,3.84) \\
0.33 \\
(-3.75,4.41) \\
0.74 \\
(-2.81,4.28) \\
\text { Sorafenib+ } \\
\text { Everolimus }\end{array}$ & $\begin{array}{c}1.24 \\
(-1.46,3.94) \\
0.41 \\
(-2.45,3.26) \\
1.44 \\
(-1.53,4.40) \\
0.06 \\
(-3.53,3.65) \\
-0.58 \\
(-3.77,2.61) \\
-5.38 \\
(-11.65,0.88) \\
-1.00 \\
(-3.64,1.63) \\
0.62 \\
(-2.11,3.34) \\
-0.33 \\
(-3.11,2.45) \\
-0.50 \\
(-3.33,2.33) \\
-1.13 \\
(-4.65,2.39) \\
-1.71 \\
(-4.58,1.17) \\
-1.72 \\
(-5.23,1.78) \\
-1.31 \\
(-4.26,1.64) \\
-2.05 \\
(-6.22,2.12) \\
\text { Bevacizumab } \\
\text { +Erlotinib }\end{array}$ \\
\hline
\end{tabular}

Values in cell are difference of median overall survival along with 95\% confidence interval.

FOLFOX4, fluorouracil, leucovorin, and oxaliplatin: PIAF, cisplatin, interferon $\alpha-2 b$, doxorubicin, and fluorouracil. 
TABLE 6 | Comparisons of median progression-free survival between treatment regimens.

\begin{tabular}{|c|c|c|c|c|c|c|c|c|c|c|c|c|c|c|c|}
\hline Placebo & $\begin{array}{c}0.27 \\
(-0.15,0.68) \\
\text { Doxorubicin }\end{array}$ & $\begin{array}{c}0.21 \\
(-0.34,0.76) \\
-0.06 \\
(-0.51,0.38) \\
\text { Nolatrexed }\end{array}$ & $\begin{array}{c}1.31 \\
(0.58,2.03) \\
1.04 \\
(0.39,1.68) \\
1.10 \\
(0.31,1.89) \\
\text { FOLFOX4 }\end{array}$ & $\begin{array}{c}3.27 \\
(1.12,5.41) \\
3.00 \\
(0.78,5.22) \\
3.06 \\
(0.79,5.34) \\
1.96 \\
(-0.38,4.30) \\
\text { Sorafenib+ } \\
\text { Doxorubicin }\end{array}$ & $\begin{array}{c}1.57 \\
(0.03,3.11) \\
1.30 \\
(-0.21,2.81) \\
1.36 \\
(-0.17,2.90) \\
0.26 \\
(-1.36,1.89) \\
-1.70 \\
(-4.28,0.89) \\
\text { Sorafenib } \\
\text { +GEMOX }\end{array}$ & $\begin{array}{c}1.09 \\
(0.77,1.41) \\
0.82 \\
(0.46,1.18) \\
0.88 \\
(0.38,1.39) \\
-0.22 \\
(-0.90,0.47) \\
-2.18 \\
(-4.32,-0.04) \\
-0.48 \\
(-1.99,1.03) \\
\text { Sorafenib }\end{array}$ & $\begin{array}{c}0.83 \\
(0.33,1.34) \\
0.56 \\
(0.05,1.08) \\
0.63 \\
(0.00,1.26) \\
-0.47 \\
(-1.25,0.31) \\
-2.43 \\
(-4.61,-0.26) \\
-0.74 \\
(-2.30,0.83) \\
-0.26 \\
(-0.66,0.14) \\
\text { Sunitinib }\end{array}$ & $\begin{array}{c}1.40 \\
(0.82,1.98) \\
1.13 \\
(0.53,1.73) \\
1.19 \\
(0.50,1.89) \\
0.09 \\
(-0.74,0.93) \\
-1.87 \\
(-4.06,0.33) \\
-0.17 \\
(-1.76,1.42) \\
0.31 \\
(-0.18,0.80) \\
0.57 \\
(-0.07,1.20) \\
\text { Brivanib }\end{array}$ & $\begin{array}{c}2.05 \\
(1.35,2.75) \\
1.78 \\
(1.07,2.49) \\
1.85 \\
(1.05,2.64) \\
0.75 \\
(-0.17,1.67) \\
-1.22 \\
(-3.47,1.04) \\
0.48 \\
(-1.16,2.12) \\
0.96 \\
(0.34,1.59) \\
1.22 \\
(0.48,1.96) \\
0.65 \\
(-0.14,1.45) \\
\text { Linifanib }\end{array}$ & $\begin{array}{c}0.58 \\
(-0.40,1.55) \\
0.31 \\
(-0.63,1.25) \\
0.37 \\
(-0.63,1.37) \\
-0.73 \\
(-1.84,0.38) \\
-2.69 \\
(-5.02,-0.36) \\
-0.99 \\
(-2.75,0.77) \\
-0.51 \\
(-1.45,0.43) \\
-0.26 \\
(-1.27,0.76) \\
-0.82 \\
(-1.89,0.25) \\
-1.48 \\
(-2.61,-0.34) \\
\text { Dovitinib }\end{array}$ & $\begin{array}{c}3.26 \\
(2.43,4.09) \\
2.99 \\
(2.15,3.83) \\
3.06 \\
(2.14,3.97) \\
1.96 \\
(0.93,2.98) \\
-0.01 \\
(-2.28,2.27) \\
1.69 \\
(-0.01,3.39) \\
2.17 \\
(1.41,2.93) \\
2.43 \\
(1.57,3.29) \\
1.86 \\
(0.97,2.76) \\
1.21 \\
(0.23,2.19) \\
2.69 \\
(1.48,3.89) \\
\text { Lenvatinib }\end{array}$ & $\begin{array}{c}0.50 \\
(-0.45,1.45) \\
0.23 \\
(-0.67,1.13) \\
0.29 \\
(-0.67,1.25) \\
-0.81 \\
(-1.87,0.25) \\
-2.77 \\
(-5.08,-0.46) \\
-1.07 \\
(-2.82,0.68) \\
-0.59 \\
(-1.53,0.35) \\
-0.34 \\
(-1.34,0.67) \\
-0.90 \\
(-1.97,0.17) \\
-1.55 \\
(-2.68,-0.43) \\
-0.08 \\
(-1.37,1.21) \\
-2.76 \\
(-3.99,-1.54) \\
\text { Nintedanib }\end{array}$ & $\begin{array}{c}0.57 \\
(0.00,1.13) \\
0.30 \\
(-0.28,0.87) \\
0.36 \\
(-0.30,1.02) \\
-0.74 \\
(-1.57,0.08) \\
-2.70 \\
(-4.88,-0.53) \\
-1.01 \\
(-2.58,0.57) \\
-0.53 \\
(-1.01,-0.04) \\
-0.27 \\
(-0.77,2.54) \\
-0.83 \\
(-1.52,-0.15) \\
-1.49 \\
(-2.28,-0.70) \\
-0.01 \\
(-1.05,1.03) \\
-2.70 \\
(-3.61,-1.79) \\
0.07 \\
(-0.97,1.10) \\
\text { Sorafenib+ } \\
\text { Erlotinib }\end{array}$ & $\begin{array}{c}1.72 \\
(0.09,3.36) \\
1.45 \\
(-0.12,3.03) \\
1.52 \\
(-0.09,3.12) \\
0.42 \\
(-1.27,2.10) \\
-1.55 \\
(-4.23,1.13) \\
0.15 \\
(-2.02,2.32) \\
0.63 \\
(-0.97,2.24) \\
0.89 \\
(-0.77,2.54) \\
0.32 \\
(-1.36,2.00) \\
-0.33 \\
(-2.06,1.40) \\
1.14 \\
(-0.68,2.97) \\
-1.54 \\
(-3.32,0.24) \\
1.22 \\
(-0.60,3.04) \\
1.16 \\
(-0.51,2.82) \\
\text { Sorafenib+ } \\
\text { Everolimus }\end{array}$ & $\begin{array}{c}2.53 \\
(0.60,4.45) \\
2.26 \\
(0.34,4.17) \\
2.32 \\
(0.35,4.29) \\
1.22 \\
(-0.77,3.21) \\
-0.74 \\
(-3.59,2.10) \\
0.95 \\
(-1.51,3.42) \\
1.43 \\
(-0.48,3.35) \\
1.69 \\
(-0.27,3.65) \\
1.12 \\
(-0.86,3.11) \\
0.47 \\
(-1.55,2.49) \\
1.95 \\
(-0.21,4.10) \\
-0.74 \\
(-2.83,1.35) \\
2.03 \\
(-0.11,4.17) \\
1.96 \\
(-0.02,3.94) \\
0.80 \\
(-1.73,3.34) \\
\text { Bevacizumab } \\
+ \text { Erlotinib }\end{array}$ \\
\hline
\end{tabular}

Values are differences of median progression-free survival along with $95 \%$ confidence interval.

FOLFOX4, fluorouracil leucovorin and oxaliplatin. GEMOX gemcitabine, oxaliplatin. 


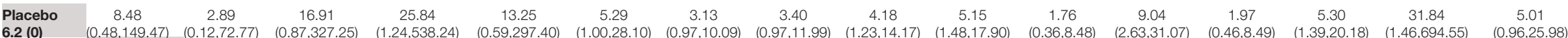

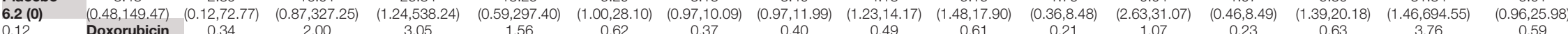

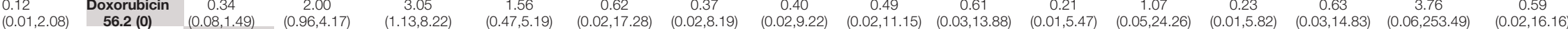

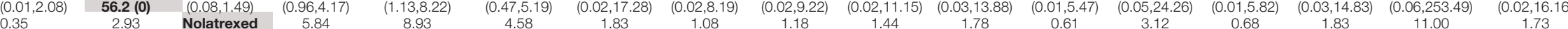

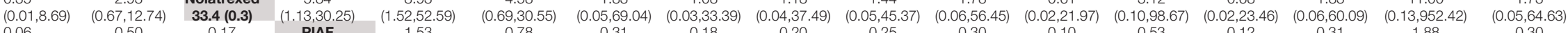

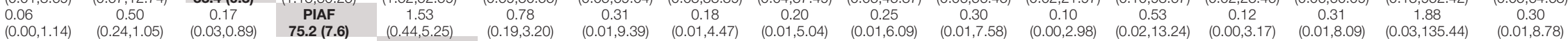

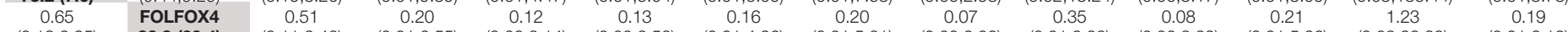

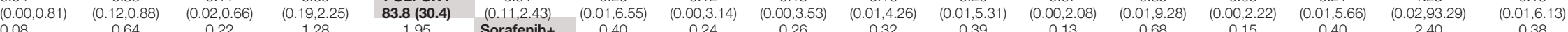

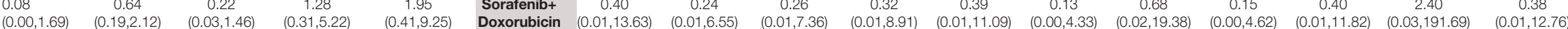

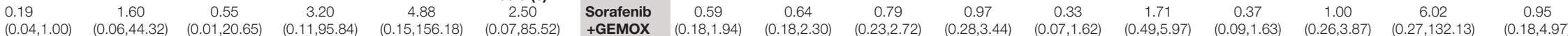

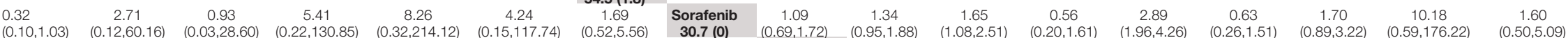

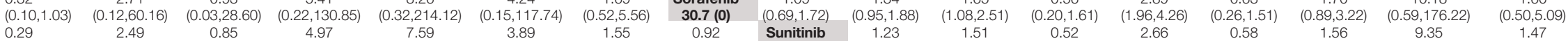

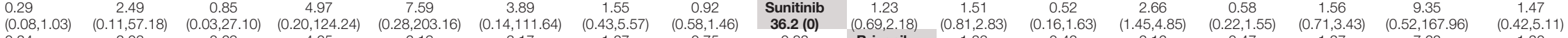

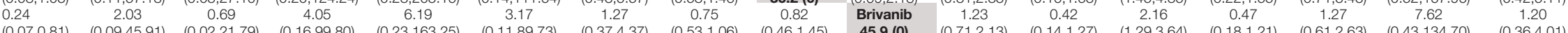

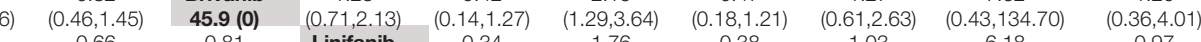

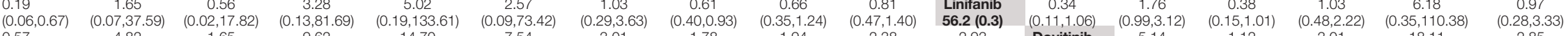

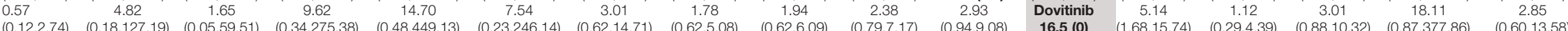

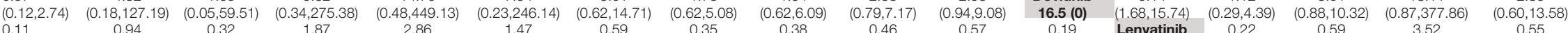

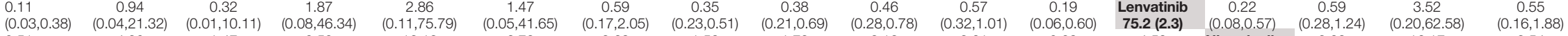

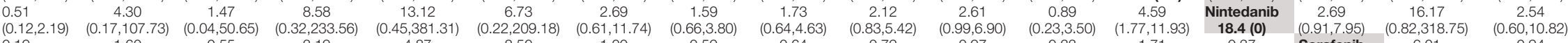

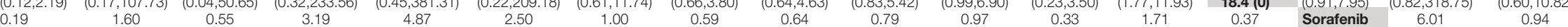

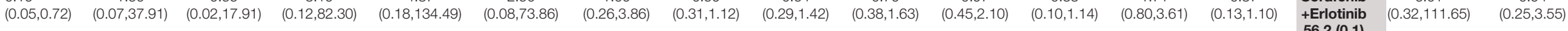

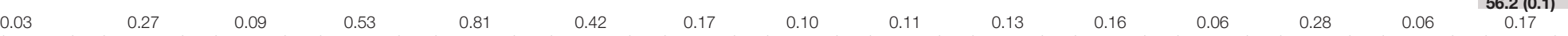
$\begin{array}{llllllllllllllll}(0.00,0.68) & (0.00,17.96) & (0.00,7.87) & (0.01,38.19) & (0.01,61.43) & (0.01,33.21) & (0.01,3.65) & (0.01,1.70) & (0.01,1.92) & (0.01,2.32) & (0.01,2.89) & (0.00,1.15) & (0.02,5.04) & (0.00,1.22) & (0.01,3.09) & \text { Everolimus }\end{array}$ $\begin{array}{llcccccccccccccc}0.20 & 1.69 & 0.58 & 3.38 & 5.16 & 2.65 & 1.06 & 0.62 & 0.68 & 0.83 & 1.03 & 0.35 & 1.81 & 0.39 & 1.06 & 6.36\end{array}$

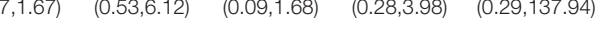


TABLE 8 | Estimations of relative treatment effects of adverse events.

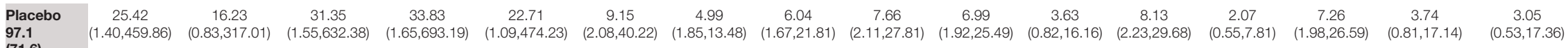

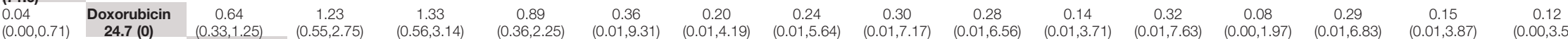

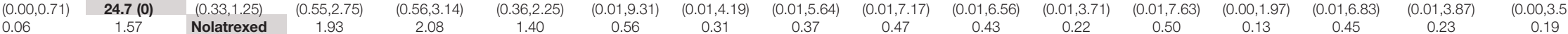

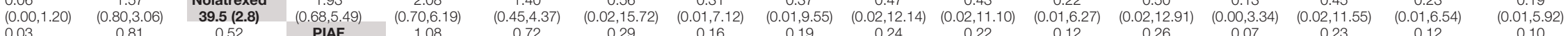

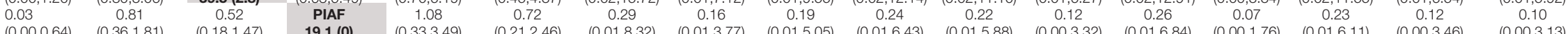

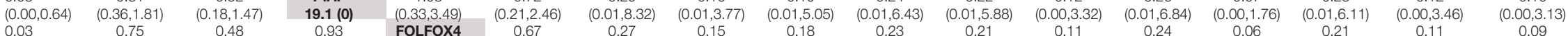

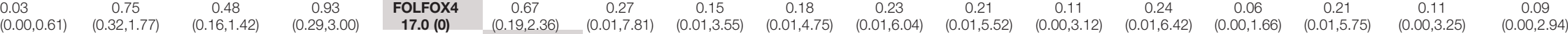

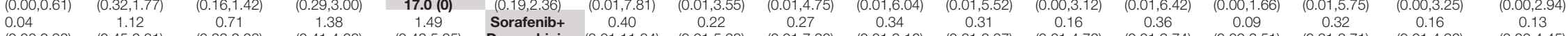

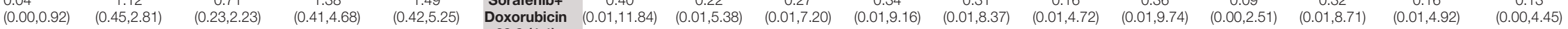

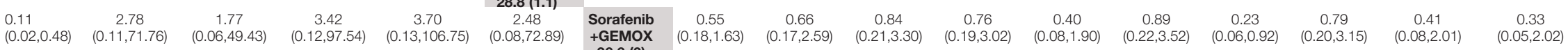

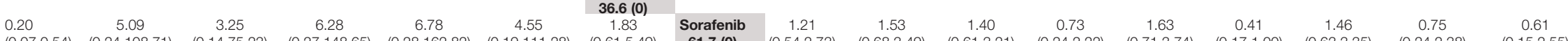

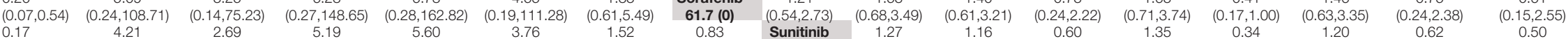

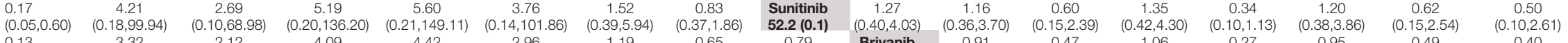

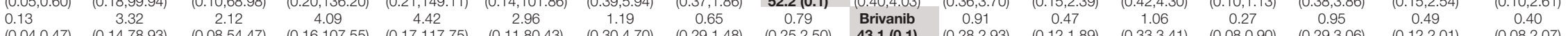

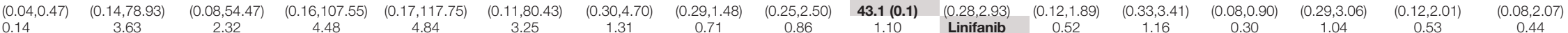

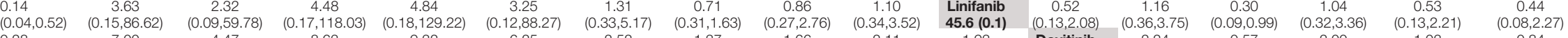

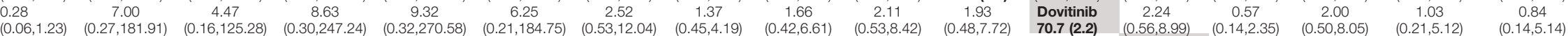

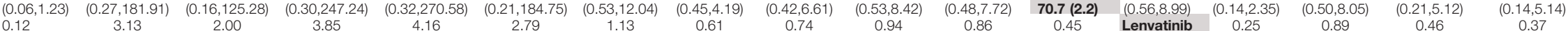

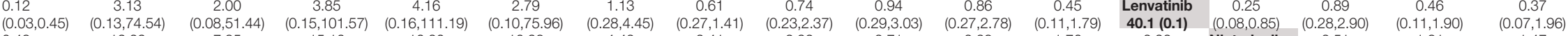

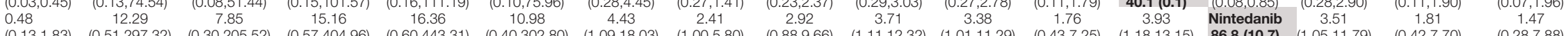

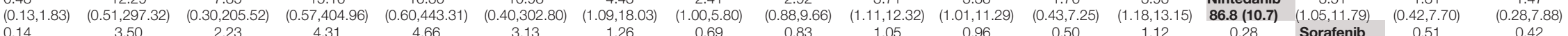

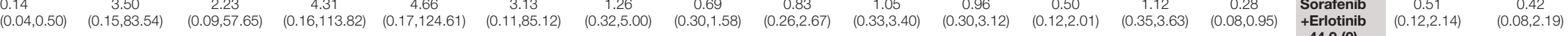

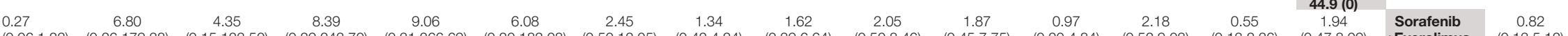
$\begin{array}{llcccccccccccccc}0.33 & 8.35 & 5.33 & 10.29 & 11.11 & 7.46 & 3.01 & 1.64 & 1.98 & 2.52 & 2.30 & 1.19 & 2.67 & 0.68 & 2.39 & 1.23\end{array}$ 
A

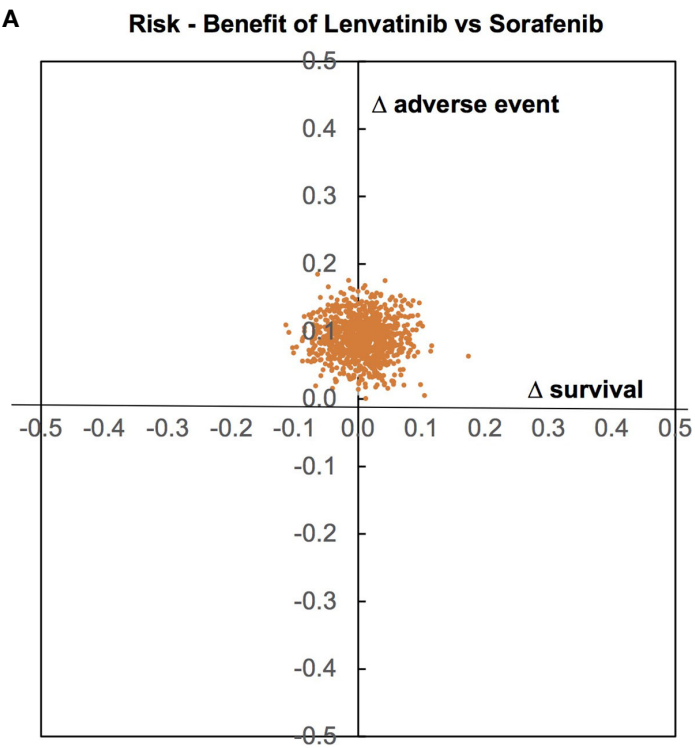

B

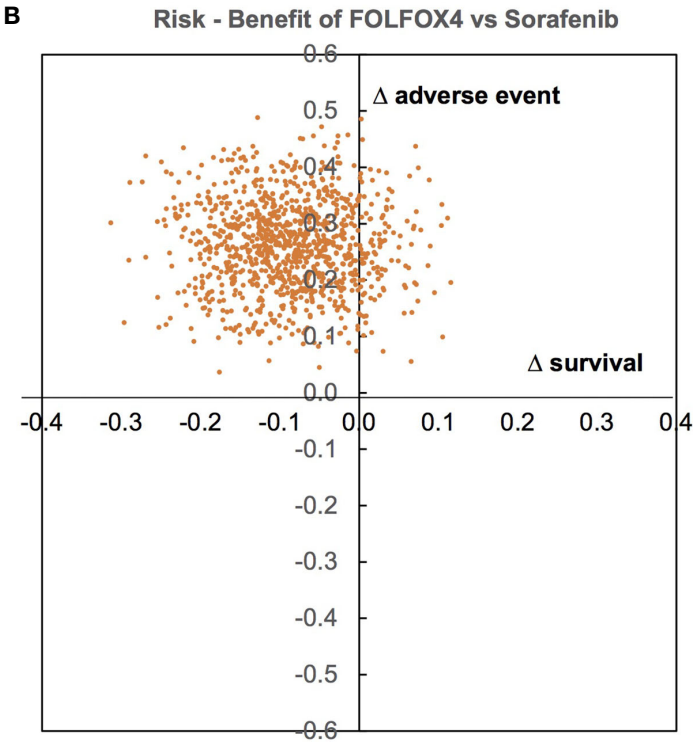

FIGURE 4 | Risk of adverse events and benefit of overall survival assessment. (A - between Lenvatinib and Sorafenib, B - between FOLFOX4 and Sorafenib).

Although our results favored MKIs as the treatment of choice in advanced HCC, many developing countries may have less access to these drugs due to high cost factors. Therefore, FOLFOX4 may still be considered as the best treatment option in these settings, as suggested by our findings. FOLFOX4 was ranked the highest among chemotherapy agents, but still showed shorter OS and higher AEs rates compared to Sorafenib and Lenvatinib.

The combination of chemotherapy and MKIs (i.e., Sorafenib + Doxorubicin or Sorafenib+GEMOX) or the combination of
MKIs (Sorafenib+Erlotinib, Sorafenib+Everolimus, or Bevacizumab+Erlotinib) may also hold some promising outcome for improving OS and PFS. However, all of the combination treatments except Sorafenib+Erlotinib were from phase II RCTs $(21,30,31,38)$, which may overestimate the efficacy due to high selection bias in those trials. Those findings require further evidence for confirmation.

Our study has some strengths. Firstly, we extracted time along with probability of OS/PFS from Kaplan-Meier curves using Digitizer software. Numbers of events (i.e., death and disease progression) along with person-time at each distinct point of the Kaplan-Meier curve were extracted. These data were then converted to individual patient data using methods suggested by Wei and Royston (26). This allowed us to be more flexible in applying a mixed-effect model (28) for time to event data analysis. Median OS and PFS were then estimated for each regimen. Among 20 RCTs, five studies did not report numbers of events and person-time at risk at each distinct time. Therefore, the estimation of hazard functions for these studies might be biased as their hazard ratios, which were estimated from individual level data, were different from their actual reported data.

Nonetheless, there were some limitations, as our review included primary studies published from 1988 to 2019. Since studies of MKIs are more recent than that of chemotherapy, their longer survival times may be confounded with improvements in supportive care over time. Recently, combination immune checkpoint inhibitor (Atezolizumab+Bevacizumab) (43) has been approved as the first-line treatment of advanced HCC and newer treatment options are still being studied in clinical trials. However, accessibility to these treatments is still limited particularly in developing countries due to their costs. Due to the high cost of treatment and grave prognosis of the disease, preventive strategy should be promoted and applied to decrease the incidence of new cases. Adjunctive treatments, which could modify tumorigenesis pathways or major risk factors of HCC such as viral hepatitis B/C infections with epigenetics, microRNAs or microenvironment modification $(44,45)$, should be explored in clinical studies to seek novel therapeutic options for improving survival and quality of life for HCC patients.

In conclusion, for limited resource countries, results from this NMA support the use of MKIs, i.e., Lenvatinib or Sorafenib, as a first-line systemic treatment for advanced HCC with preserved liver function (Child-Pugh A) and non-significant portal vein involvement. FOLFOX4, chemotherapy, might be an option if MKIs are less accessible. This NMA should be updated when more phase-III RCTs are published.

\section{DATA AVAILABILITY STATEMENT}

The original contributions presented in the study are included in the article/supplementary material. Further inquiries can be directed to the corresponding author. 


\section{AUTHOR CONTRIBUTIONS}

SO: Conceptualization, Methodology, Formal analysis, Investigation, Writing-Original draft preparation. AP and ATa: Validation. SR and TR: Supervision. ATh: Writing-Review and editing, Supervision. JA: Writing-Review and editing. All authors contributed to the article and approved the submitted version.

\section{REFERENCES}

1. Sung H, Ferlay J, Siegel RL, Laversanne M, Soerjomataram I, Jemal A, et al. Global cancer statistics 2020: GLOBOCAN estimates of incidence and mortality worldwide for 36 cancers in 185 countries. CA Cancer J Clin (2021). doi: 10.3322/caac. 21660

2. Llovet JM, Bru C, Bruix J. Prognosis of hepatocellular carcinoma: the BCLC staging classification. Semin Liver Dis (1999) 19(3):329-38. doi: 10.1055/s2007-1007122

3. Marrero JA, Kulik LM, Sirlin CB, Zhu AX, Finn RS, Abecassis MM, et al. Diagnosis, Staging, and Management of Hepatocellular Carcinoma: 2018 Practice Guidance by the American Association for the Study of Liver Diseases. Hepatology (2018) 68(2):723-50. doi: 10.1002/hep.29913

4. Llovet JM, Ricci S, Mazzaferro V, Hilgard P, Gane E, Blanc JF, et al. Sorafenib in advanced hepatocellular carcinoma. N Engl J Med (2008) 359(4):378-90. doi: 10.1056/NEJMoa0708857

5. Johnson PJ, Qin S, Park JW, Poon RT, Raoul JL, Philip PA, et al. Brivanib versus sorafenib as first-line therapy in patients with unresectable, advanced hepatocellular carcinoma: results from the randomized phase III BRISK-FL study. J Clin Oncol (2013) 31(28):3517-24. doi: 10.1200/jco.2012.48.4410

6. Cheng AL, Kang YK, Lin DY, Park JW, Kudo M, Qin S, et al. Sunitinib versus sorafenib in advanced hepatocellular cancer: results of a randomized phase III trial. J Clin Oncol (2013) 31(32):4067-75. doi: 10.1200/jco.2012.45.8372

7. Cainap C, Qin S, Huang WT, Chung IJ, Pan H, Cheng Y, et al. Linifanib versus Sorafenib in patients with advanced hepatocellular carcinoma: results of a randomized phase III trial. J Clin Oncol (2015) 33(2):172-9. doi: 10.1200/ jco.2013.54.3298

8. Kudo M, Finn RS, Qin S, Han KH, Ikeda K, Piscaglia F, et al. Lenvatinib versus sorafenib in first-line treatment of patients with unresectable hepatocellular carcinoma: a randomised phase 3 non-inferiority trial. Lancet (2018) 391(10126):1163-73. doi: 10.1016/S0140-6736(18)30207-1

9. Cucchetti A, Piscaglia F, Pinna AD, Djulbegovic B, Mazzotti F, Bolondi L. Efficacy and Safety of Systemic Therapies for Advanced Hepatocellular Carcinoma: A Network Meta-Analysis of Phase III Trials. Liver Cancer (2017) 6(4):337-48. doi: 10.1159/000481314

10. McNamara MG, Slagter AE, Nuttall C, Frizziero M, Pihlak R, Lamarca A, et al. Sorafenib as first-line therapy in patients with advanced Child-Pugh B hepatocellular carcinoma-a meta-analysis. Eur J Cancer (2018) 105:1-9. doi: 10.1016/j.ejca.2018.09.031

11. Peng S, Zhao Y, Xu F, Jia C, Xu Y, Dai C. An updated meta-analysis of randomized controlled trials assessing the effect of sorafenib in advanced hepatocellular carcinoma. PloS One (2014) 9(12):e112530. doi: 10.1371/ journal.pone.0112530

12. Petrelli F, Coinu A, Borgonovo K, Cabiddu M, Ghilardi M, Lonati V, et al. Oxaliplatin-based Chemotherapy: A New Option in Advanced Hepatocellular Carcinoma. A Systematic Review and Pooled Analysis. Clin Oncol (2014) 26 (8):488-96. doi: 10.1016/j.clon.2014.04.031

13. Shao YY, Shau WY, Chan SY, Lu LC, Hsu CH, Cheng AL. Treatment efficacy differences of sorafenib for advanced hepatocellular carcinoma: A metaanalysis of randomized clinical trials. Oncology (Switzerland) (2015) 88 (6):345-52. doi: 10.1159/000369559

14. Shen A, Tang C, Wang Y, Chen Y, Yan X, Zhang C, et al. A systematic review of sorafenib in child-pugh A patients with unresectable hepatocellular carcinoma. J Clin Gastroenterol (2013) 47(10):871-80. doi: 10.1097/ MCG.0b013e3182a87cfd

15. Wang $\mathrm{H}$, Wang $\mathrm{H}, \mathrm{Yu} \mathrm{Z}$, Liu H. Alternative treatment strategies to sorafenib in patients with advanced hepatocellular carcinoma: A meta-analysis of

\section{ACKNOWLEDGMENTS}

This manuscript is a part of SO's thesis of international Ph.D. program (Clinical Epidemiology), at the Department of Clinical Epidemiology and Biostatistics, Faculty of Medicine, Ramathibodi Hospital, Mahidol University, Bangkok, Thailand. The authors would like to thank Mr. Tim Bagwell and Mr. Stephen Pinder for English editing.

randomized phase iii trials. Onco Targets Ther (2018) 11:5195-201. doi: 10.2147/OTT.S171918

16. Zhang X, Yang XR, Huang XW, Wang WM, Shi RY, Xu Y, et al. Sorafenib in treatment of patients with advanced hepatocellular carcinoma: A systematic review. Hepatobiliary Pancreat Dis Int (2012) 11(5):458-66. doi: 10.1016/ S1499-3872(12)60209-4

17. Wang Z, Wu XL, Zeng WZ, Xu GS, Xu H, Weng M, et al. Meta-analysis of the efficacy of sorafenib for hepatocellular carcinoma. Asian Pac J Cancer Prev (2013) 14(2):691-4. doi: 10.7314/apjcp.2013.14.2.691

18. Cheng AL, Kang YK, Chen Z, Tsao CJ, Qin S, Kim JS, et al. Efficacy and safety of sorafenib in patients in the Asia-Pacific region with advanced hepatocellular carcinoma: a phase III randomised, double-blind, placebocontrolled trial. Lancet Oncol (2009) 10(1):25-34. doi: 10.1016/S1470-2045 (08)70285-7

19. Zhu AX, Rosmorduc O, Evans TR, Ross PJ, Santoro A, Carrilho FJ, et al. SEARCH: a phase III, randomized, double-blind, placebo-controlled trial of sorafenib plus erlotinib in patients with advanced hepatocellular carcinoma. J Clin Oncol (2015) 33(6):559-66. doi: 10.1200/jco.2013.53.7746

20. Cheng AL, Thongprasert S, Lim HY, Sukeepaisarnjaroen W, Yang TS, Wu $\mathrm{CC}$, et al. Randomized, open-label phase 2 study comparing frontline dovitinib versus sorafenib in patients with advanced hepatocellular carcinoma. Hepatology (2016) 64(3):774-84. doi: 10.1002/hep.28600

21. Koeberle D, Dufour JF, Demeter G, Li Q, Ribi K, Samaras P, et al. Sorafenib with or without everolimus in patients with advanced hepatocellular carcinoma (HCC): A randomized multicenter, multinational phase II trial (SAKK 77/08 and SASL 29). Ann Oncol (2016) 27(5):856-61. doi: 10.1093/ annonc/mdw054

22. World Health Organization. WHO handbook for reporting results of cancer treatment. Geneva: World Health Organization (1979).

23. Eisenhauer EA, Therasse P, Bogaerts J, Schwartz LH, Sargent D, Ford R, et al. New response evaluation criteria in solid tumours: revised RECIST guideline (version 1.1). Eur J Cancer (2009) 45(2):228-47. doi: 10.1016/j.ejca.2008.10.026

24. National Cancer I. Common Terminology Criteria for Adverse Events (CTCAE) v5.0. (2017). https://ctep.cancer.gov/protocolDevelopment/ electronic_applications/docs/CTCAE_v5_Quick_Reference_5x7.pdf. Accessed July 7, 2020.

25. Drevon D, Fursa SR, Malcolm AL. Intercoder Reliability and Validity of WebPlotDigitizer in Extracting Graphed Data. Behav Modif (2017) 41(2):32339. doi: $10.1177 / 0145445516673998$

26. Wei Y, Royston P. Reconstructing time-to-event data from published KaplanMeier curves. Stata J (2017) 17(4):786-802. doi: 10.1177/1536867X1701700402

27. Sterne JAC, Savovic J, Page MJ, Elbers RG, Blencowe NS, Boutron I, et al. RoB 2: a revised tool for assessing risk of bias in randomised trials. $B M J$ (2019) 366: 14898. doi: 10.1136/bmj.14898

28. Crowther MJ. Multilevel mixed-effects parametric survival analysis: Estimation, simulation, and application. Stata J (2019) 19(4):931-49. doi: 10.1177/1536867X19893639

29. StataCrop. Stata Statistical Software: Release 16. Collage Station, TX: StataCorp LLC (2019).

30. Abou-Alfa GK, Johnson P, Knox JJ, Capanu M, Davidenko I, Lacava J, et al. Doxorubicin plus sorafenib vs doxorubicin alone in patients with advanced hepatocellular carcinoma: A randomized trial. JAMA - J Am Med Assoc (2010) 304(19):2154-60. doi: 10.1001/jama.2010.1672

31. Assenat E, Pageaux GP, Thézenas S, Peron JM, Bécouarn Y, Seitz JF, et al. Sorafenib alone vs. sorafenib plus GEMOX as 1 st -line treatment for advanced HCC: the phase II randomised PRODIGE 10 trial. Br J Cancer (2019) 120 (9):896-902. doi: 10.1038/s41416-019-0443-4 
32. Gish RG, Porta C, Lazar L, Ruff P, Feld R, Croitoru A, et al. Phase III randomized controlled trial comparing the survival of patients with unresectable hepatocellular carcinoma treated with nolatrexed or doxorubicin. J Clin Oncol (2007) 25 (21):3068-75. doi: 10.1200/JCO.2006.08.4046

33. Ji YX, Zhang ZF, Lan KT, Nie KK, Geng CX, Liu SC, et al. Sorafenib in liver function impaired advanced hepatocellular carcinoma. Chin Med Sci J (2014) 29(1):7-14. doi: 10.1016/S1001-9294(14)60017-1

34. Lai CL, Lok AS, Wu PC, Chan GC, Lin HJ. Doxorubicin versus no antitumor therapy in inoperable hepatocellular carcinoma. A prospective randomized trial. Cancer (1988) 62(3):479-83. doi: 10.1002/1097-0142(19880801) 62:3<479::AID-CNCR2820620306>3.0.CO;2-L

35. Mok TSK, Leung TWT, Lee SD, Chao Y, Chan ATC, Huang A, et al. A multicentre randomized phase II study of nolatrexed versus doxorubicin in treatment of Chinese patients with advanced hepatocellular carcinoma. Cancer Chemother Pharmacol (1999) 44(4):307-11. doi: 10.1007/s002800050982

36. Palmer DH, Ma YT, Peck-Radosavljevic M, Ross P, Graham J, Fartoux L, et al. A multicentre, open-label, phase-I/randomised phase-II study to evaluate safety, pharmacokinetics, and efficacy of nintedanib vs. sorafenib in European patients with advanced hepatocellular carcinoma. Br J Cancer (2018) 118 (9):1162-8. doi: 10.1038/s41416-018-0051-8

37. Qin S, Bai Y, Lim HY, Thongprasert S, Chao Y, Fan J, et al. Randomized, multicenter, open-label study of oxaliplatin plus fluorouracil/leucovorin versus doxorubicin as palliative chemotherapy in patients with advanced hepatocellular carcinoma from Asia. J Clin Oncol (2013) 31(28):3501-8. doi: 10.1200/JCO.2012.44.5643

38. Thomas MB, Garrett-Mayer E, Anis M, Anderton K, Bentz T, Edwards A, et al. A Randomized Phase II Open-Label Multi-Institution Study of the Combination of Bevacizumab and Erlotinib Compared to Sorafenib in the First-Line Treatment of Patients with Advanced Hepatocellular Carcinoma. Oncology (Switzerland) (2018) 94(6):329-39. doi: 10.1159/000485384

39. Yen CJ, Kim TY, Feng YH, Chao Y, Lin DY, Ryoo BY, et al. A Phase I/ Randomized Phase II Study to Evaluate the Safety, Pharmacokinetics, and Efficacy of Nintedanib versus Sorafenib in Asian Patients with Advanced Hepatocellular Carcinoma. Liver Cancer (2018) 7(2):165-78. doi: 10.1159/ 000486460
40. Yeo W, Mok TS, Zee B, Leung TWT, Lai PBS, Lau WY, et al. A randomized phase III study of doxorubicin versus cisplatin/interferon $\alpha$-2b/doxorubicin/ fluorouracil (PIAF) combination chemotherapy for unresectable hepatocellular carcinoma. J Natl Cancer Inst (2005) 97(20):1532-8. doi: 10.1093/jnci/dji315

41. NCCN Clinical Practice Guidelines in Oncology (NCCN Guidelines ${ }^{\circledR}$ ) Hepatobiliary Cancers version 4.2019. (2019). https://www.nccn.org/ professionals/physician_gls/pdf/hepatobiliary.pdf [Accessed December 20, 2019].

42. Liu L, Zheng YH, Han L, Qin SK. Efficacy and safety of the oxaliplatin-based chemotherapy in the treatment of advanced primary hepatocellular carcinoma: A meta-analysis of prospective studies. Medicine (Baltimore) (2016) 95(40):e4993. doi: 10.1097/MD.0000000000004993

43. Finn RS, Qin S, Ikeda M, Galle PR, Ducreux M, Kim TY, et al. Atezolizumab plus Bevacizumab in Unresectable Hepatocellular Carcinoma. N Engl J Med (2020) 382(20):1894-905. doi: 10.1056/NEJMoa1915745

44. Gnoni A, Santini D, Scartozzi M, Russo A, Licchetta A, Palmieri V, et al. Hepatocellular carcinoma treatment over sorafenib: epigenetics, microRNAs and microenvironment. Is there a light at the end of the tunnel? Expert Opin Ther Targets (2015) 19(12):1623-35. doi: 10.1517/14728222.2015.1071354

45. D’Anzeo M, Faloppi L, Scartozzi M, Giampieri R, Bianconi M, Del Prete M, et al. The role of micro-RNAs in hepatocellular carcinoma: from molecular biology to treatment. Molecules (2014) 19(5):6393-406. doi: 10.3390/molecules 19056393

Conflict of Interest: The authors declare that the research was conducted in the absence of any commercial or financial relationships that could be construed as a potential conflict of interest.

Copyright (C) 2021 Oranratnachai, Rattanasiri, Pooprasert, Tansawet, Reungwetwattana, Attia and Thakkinstian. This is an open-access article distributed under the terms of the Creative Commons Attribution License (CC BY). The use, distribution or reproduction in other forums is permitted, provided the original author(s) and the copyright owner(s) are credited and that the original publication in this journal is cited, in accordance with accepted academic practice. No use, distribution or reproduction is permitted which does not comply with these terms. 